TRANSACTIONS OF THE

AMERICAN MATHEMATICAL SOCIETY

Volume 359, Number 6, June 2007, Pages 2881-2898

S 0002-9947(07)04108-6

Article electronically published on January 26, 2007

\title{
DIMENSIONAL PROPERTIES OF THE HARMONIC MEASURE FOR A RANDOM WALK ON A HYPERBOLIC GROUP
}

\author{
VINCENT LE PRINCE
}

\begin{abstract}
This paper deals with random walks on isometry groups of Gromov hyperbolic spaces, and more precisely with the dimension of the harmonic measure $\nu$ associated with such a random walk. We first establish a link of the form $\operatorname{dim} \nu \leq h / l$ between the dimension of the harmonic measure, the asymptotic entropy $h$ of the random walk and its rate of escape $l$. Then we use this inequality to show that the dimension of this measure can be made arbitrarily small and deduce a result on the type of the harmonic measure.
\end{abstract}

\section{INTRODUCTION}

Let $(X, d)$ be a hyperbolic space, equipped with a base point $o$, and $G$ a nonelementary subgroup of its isometry group acting properly discontinuously. Given a probability measure $\mu$ on $G$, we define the associated random walk $\left(x_{n}\right)$ by $x_{n}=$ $h_{1} \cdots h_{n},\left(h_{i}\right)$ being a sequence of $\mu$-distributed independent random variables. We can describe the asymptotic behaviour of this random walk ([13]): if the support of $\mu$ generates $G$, then the trajectory $\left(x_{n} o\right)$ converges almost surely to an element $x_{\infty}$ of the hyperbolic boundary $\partial X$ of $X$, whose distribution is called the harmonic measure and denoted by $\nu$. If moreover $\mu$ has a finite first moment, then $(\partial X, \nu)$ coincides with the Poisson boundary of the random walk ([13), see Section 1 for the definitions and details).

On the boundary of a tree we can define a metric by the formula:

$$
\operatorname{dist}\left(\xi_{1}, \xi_{2}\right)=e^{-\left(\xi_{1} \mid \xi_{2}\right)},
$$

$\left(\xi_{1} \mid \xi_{2}\right)$ being the Gromov product (here the length of the initial common part) of $\xi_{1}$ and $\xi_{2}$. In the general case, this formula doesn't define a metric, but there exists a family of metrics $\left(d_{a}\right)_{1<a<a_{0}}$ on $\partial X$ having similar properties. We shall consider in this article the relations between this metric structure and the harmonic measure. More precisely we are interested in the Hausdorff dimension of this measure (see Section 1 for a definition) and consequently in its type. Our main result will be the fact that the harmonic measure is not necessarily absolutely continuous with respect to the Hausdorff measure on the boundary but can be singular. This question has been considered for random walks on $S L(2, \mathbb{R})$ related to continued fractions ([2], [16]) and in some other contexts (e.g. [22]).

Received by the editors December 16, 2004 and, in revised form, June 17, 2005.

2000 Mathematics Subject Classification. Primary 60G50, 20F67, 28D20, 28A78.

Key words and phrases. Ergodic theory, random walk, hyperbolic group, harmonic measure, entropy.

(C)2007 American Mathematical Society Reverts to public domain 28 years from publication 
Note that in some situations, such as for the nearest neighbor random walk on finitely generated free groups (see [7, [19, [18]) or on finite free products of finite groups ([20]), this harmonic measure can be explicitly computed, but in general this is not the case. Whereas in the above cited articles combinatorial methods, based on a description of the hyperbolic boundary as a set of infinite words, are used, we shall use geometrical methods.

Let us now describe more precisely the contents of this paper.

The following relation between the dimension of $\nu$, the rate of escape $l$ of the random walk and its asymptotic entropy $h$ (see Section 1 for the definitions) was established in the case of trees in [12] and [18]:

$$
\operatorname{dim} \nu=\frac{h}{l} .
$$

Section 2 is devoted to an extension of this result to the case of a subgroup of the group of isometries of a hyperbolic space (see Proposition 2.3 for a more precise statement):

$$
\operatorname{dim} \nu \leq \frac{1}{\log a} \frac{h}{l},
$$

$a$ being the parameter used for the choice of the metric on the hyperbolic boundary. Then, in order to show that the dimension of this measure can be arbitrarily small, we construct in Section 4 a sequence $\left(\mu_{k}\right)$ of probability measures such that, denoting the asymptotic entropy and the rate of escape associated with $\mu_{k}$ respectively by $h\left(G, \mu_{k}\right)$ and $l\left(G, \mu_{k}\right)$, we have

$$
\lim _{k \rightarrow+\infty} \frac{h\left(G, \mu_{k}\right)}{l\left(G, \mu_{k}\right)}=0 .
$$

In order to prove this property we show in Section 3 a formula which allows us to estimate the rate of escape. The association of inequality (0.2) and identity (0.3) gives us the following result.

Theorem 5.1. Let $G$ be a subgroup of the group of isometries of a hyperbolic space $(X, d)$ acting properly discontinuously and which is not elementary. For every $\epsilon>0$ there exists on $G$ a symmetric probability measure $\mu$ with a finite first moment, whose support generates $G$, and which has the following property: the pointwise dimension of the harmonic measure $\nu$ associated with $(G, \mu)$ is $\nu$-almost surely smaller than $\epsilon$.

In the case where $X$ is the Cayley graph of the hyperbolic group $G$ equipped with the word metric (w.r.t. a certain system of generators $S$ ), the dimension of the boundary $\partial G$ is equal to the growth $v(G, S)$ of $G$ (w.r.t. the same $S$ ). Moreover the Hausdorff measure is then finite and nonzero. Theorem 5.1 implies that the harmonic measure can be singular with respect to this Hausdorff measure (Corollary $5.3)$.

There are two other natural questions concerning the dimension of the harmonic measure. The first, which in some sense is the opposite to the one we consider, is the question of knowing whether the harmonic measure can be of maximal dimension. In the case where $X$ is the Cayley graph of $G$, the support of the harmonic measure is the boundary $\partial G$. The dimension of $\partial G$ is, denoting by $v$ the growth of $G$ and working with the distance $d_{a}$ on $\partial G$, equal to $v_{a}(G)=v / \log a$ (4), see Section 1). 
We have in this context a fundamental relation between $h, l$ and $v$ (see [23]):

$$
h \leq l v \text {. }
$$

In view of inequality (0.2), the question of the maximality of the dimension of the harmonic measure is then related to the question raised in 23 of knowing whether the quotient $h / l$ can be maximal, i.e. equal to $v$.

The second is the question of the positivity of the dimension of the harmonic measure. Under the hypotheses stated in the first paragraph of this introduction, the Poisson boundary is not trivial. A well-known fact of the theory of Poisson boundaries is that this non-triviality is equivalent to the asymptotic entropy $h$ being strictly positive ([14). So in the case of a tree the formula $\operatorname{dim} \nu=h / l$ implies that the dimension of the harmonic measure is strictly positive. But with only the inequality (0.2) we can't reach a conclusion. In [15] a similar result (i.e. positivity of the dimension of the harmonic measure) is proven in the case of the Brownian motion on a Riemannian manifold with pinched negative sectional curvature.

\section{Preliminaries and notation}

1.1. Hyperbolic spaces. We will need a rather complete description of some aspects of the geometry of hyperbolic spaces $([10])$, which we recall there. Our main references are [5] and [9].

Definition. Let $(X, d)$ be a proper geodesic metric space. Let $o$ be a point in $X$; the Gromov product (w.r.t. o) on $X^{2}$ is defined by

$$
(x \mid y)_{o}=\frac{1}{2}[d(o, x)+d(o, y)-d(x, y)] .
$$

We call $(X, d)$ a $\delta$-hyperbolic space if this product satisfies, for every $x, y, z, o$ in $X$,

$$
(x \mid z)_{o} \geq \min \left\{(x \mid y)_{o},(y \mid z)_{o}\right\}-\delta .
$$

We say that $X$ is hyperbolic if there exists a $\delta$ such that $X$ is $\delta$-hyperbolic. An important class of hyperbolic spaces is the one of word hyperbolic groups. One can define on the Cayley graph of a finitely generated group $G$ (w.r.t. a symmetric system of generators $S$ ) a metric which is the word metric (w.r.t. $S$ ) in restriction to $G$ and which makes every edge isometric to the segment $[0,1]$. A finitely generated group $G$ is said to be hyperbolic when its Cayley graph equipped with this metric is a hyperbolic space (which doesn't depend on the choice of $S$ ).

Notation. In the sequel $(X, d)$ will denote a $\delta$-hyperbolic space equipped with a point $o$, and the Gromov product of $x$ and $y$ w.r.t. $o$ will be denoted by $(x \mid y)$.

Hyperbolic boundary. We recall that a geodesic (resp. geodesic ray, resp. geodesic segment) in $X$ is an isometry from $\mathbb{R}$ (resp. $[0, \infty[$, resp. $[a, b]$ ) to $X$, as well as the image of such an isometry.

Two geodesic rays $\sigma_{1}$ and $\sigma_{2}$ in $X$ are said to be equivalent $\left(\sigma_{1} \sim \sigma_{2}\right)$ if there exists a constant $D$ such that for every $t, d\left(\sigma_{1}(t), \sigma_{2}(t)\right) \leq D$. The hyperbolic boundary is defined as the quotient of the set of geodesic rays by this equivalence relation.

One can extend the Gromov product to $X \cup \partial X$. The relation (1.1) remains true. One can then define a topology on $X \cup \partial X$, which makes $X \cup \partial X$ be a 
compactification of $X$, by taking for each point $\xi$ in $\partial X$ as a base of neighborhoods the sets

$$
\{x \in X \cup \partial X:(\xi \mid x)>R\} \quad(R>0) .
$$

A sequence $\left(x_{n}\right)$ converges to an element $\xi$ in $\partial X$ if and only if

$$
\lim _{n \rightarrow \infty}\left(\xi \mid x_{n}\right)=+\infty .
$$

In particular, if $\sigma$ is a geodesic ray, $\sigma(t)$ converges, when $t$ goes to infinity, to the equivalence class of $\sigma$ in $\partial X$, which we denote by $\sigma(+\infty)$. Moreover, if $\left(x_{n}\right)$ converges to $x$ and $\left(y_{n}\right)$ to $y$, we have

$$
(x \mid y) \leq \liminf _{n}\left(x_{n} \mid y_{n}\right) \leq(x \mid y)+2 \delta .
$$

The action of the isometry group on $X$ extends to a continuous action on the boundary.

When $X$ is the Cayley graph of a hyperbolic group $G$, we shall write $\partial G$ instead of $\partial X$.

Metric on the boundary. If $\mathrm{X}$ is a tree we can define a metric on $\partial X$ by

$$
\operatorname{dist}\left(\xi_{1}, \xi_{2}\right)=e^{-\left(\xi_{1} \mid \xi_{2}\right)} .
$$

In the general case, this formula doesn't define a metric, but there exists a family of metrics $\left(d_{a}\right)_{1<a<a_{0}}$ on $X \cup \partial X$ (see [5], ch. 11). We fix such an $a$ for the sequel. We will need the following property, which shows the analogy with the case of a tree (see [5]):

Proposition 1.1. There exists a constant $\lambda>1$ such that

- $\forall x, y \in X \cup \partial X, d_{a}(x, y) \leq \lambda a^{-(x \mid y)}$

- $\forall x, y \in \partial X, d_{a}(x, y) \geq \lambda^{-1} a^{-(x \mid y)}$.

Note that the topology induced by this metric on $X \cup \partial X$ coincides with the one defined above.

Limit set of a subgroup of isometries. Let $G$ a subgroup of isometries acting properly discontinuously on $(X, d)$. A reference for this part is 3 .

Definition 1.2. The set of accumulation points in $\partial X$ of the $G$-orbit of a point $x$ in $X$ doesn't depend on the point $x$. We call it the limit set of the group $G$ denoted by $\Lambda_{G}$.

In the case of a hyperbolic group $G$ acting on its Cayley graph, $\Lambda_{G}=\partial G$. This limit set will be the support of the harmonic measure; we shall need some of its properties.

Definition 1.3. The group $G$ is said to be elementary if $\Lambda_{G}$ consists of at most two elements.

If $G$ is non-elementary, then $\Lambda_{G}$ is uncountable.

Definition 1.4. Let $g$ be an isometry in $X$. This element is said to be hyperbolic if $\left(g^{n} o\right)_{n \in \mathbb{Z}}$ is a quasi-geodesic, which means that there exist two constants $\lambda$ and $c$ such that for all $n$ and $m$ in $\mathbb{Z}$,

$$
\lambda^{-1}|n-m|-c \leq d\left(g^{n} o, g^{m} o\right) \leq \lambda|n-m|+c .
$$


Proposition 1.5. Let $\eta$ be a $(\lambda, c)$-quasi-geodesic. Then $\eta(t)$ admits limits $\eta( \pm \infty)$ when $t$ goes to $\pm \infty$. If $\sigma$ is a geodesic with endpoints $\sigma( \pm \infty)=\eta( \pm \infty)$, then the images of $\sigma$ and $\eta$ lie at a finite Hausdorff distance $K$ from each other, $K$ depending only on $\lambda, c$ and $\delta$.

In particular if an isometry $g$ is hyperbolic, then there exists $g^{+}$and $g^{-}$in $\partial X$ such that $\left(g^{n} o\right)$ goes to $g^{+}$and $g^{-}$when $n$ goes respectively to plus or minus infinity. In fact for every $x$ in $X$, the sequence $\left(g^{n} x\right)$ converges to the same limit. The points $g^{+}$and $g^{-}$are fixed points for $g$, which are said to be respectively attractive and repulsive.

Proposition 1.6 ([3]). A non-elementary group $G$ contains hyperbolic elements.

An important property of the limit set is its minimality (see [3]).

Proposition 1.7 (Gromov). Assume that $G$ is non-elementary. Then every nonvoid $G$-invariant compact set in $\partial X$ contains $\Lambda_{G}$. In other words, $\Lambda_{G}$ is the only minimal set.

The minimality of $\Lambda_{G}$ has the following consequence:

Proposition 1.8. Assume that $G$ is non-elementary. Let $U$ be an open set in $\partial X$ which intersects $\Lambda_{G}$. Then we have

$$
\partial X=\bigcup_{g \in G} g U .
$$

Since $\partial X$ is compact, there exists a finite set of elements $g_{1}, \cdots, g_{r}$ in $G$ such that

$$
\partial X=\bigcup_{1 \leq i \leq r} g_{i} U
$$

\subsection{Random walk.}

Notation. In the sequel $G$ will denote a subgroup of the group of isometries of $(X, d)$ acting properly discontinuously and which is non-elementary.

Definition. Let $\mu$ be a probability measure on $G$. The random walk on $G$ determined by the measure $\mu$ is the Markov chain $\boldsymbol{x}=\left(x_{n}\right)_{n \geq 0}$ with transition probabilities

$$
p(x, y)=\mu\left(x^{-1} y\right)
$$

and starting at $x_{0}=e$. The position $x_{n}$ at time $n \geq 1$ of the random walk is given by

$$
x_{n}=h_{1} \cdots h_{n},
$$

$\left(h_{n}\right)_{n \geq 1}$ being a sequence of independent $\mu$-distributed random variables. We call the $h_{n}$ the increments and $\left(x_{n}\right)$ the trajectory of the random walk. We denote by $\mathbb{P}$ the distribution of $\boldsymbol{x}$ in $G^{\mathbb{N}}$ and by $T$ the shift in $G^{\mathbb{N}}$.

The Markov operator $P$ associated to the random walk is then

$$
P f(x)=\sum_{g \in G} \mu(g) f(x g) .
$$

We say that a function $f$ on $G$ is $\mu$-harmonic if it satisfies $P f=f$. 
Poisson boundary and harmonic measure. The behaviour of the paths of the random walk is described by the following:

Proposition 1.9 ([13]). Assume that the support of the measure $\mu$ generates $G$. Then the random walk $\left(x_{n} o\right)$ associated to $\mu$ converges $\mathbb{P}$-almost surely to an element $x_{\infty}$ in $\partial X$.

We denote by bnd the map (defined on a set of $\mathbb{P}$-measure 1) from $G^{\mathbb{N}}$ to $\partial X$ which associates $x_{\infty}$ to $\boldsymbol{x}=\left(x_{n}\right)$, and by $\nu$ the distribution of $x_{\infty}$, which we call the harmonic measure. Note that we have $\nu=b n d(\mathbb{P})$ and that $b n d$ is $G$-equivariant.

Let us recall that if $G$, equipped with a measure $\mu$, acts on a space $Y$ with a measure $m$, the convolution $\mu * m$ of $m$ by $\mu$ is defined by: for every continuous bounded function $f$,

$$
\int_{Y} f(y) d(\mu * m)(y)=\int_{G \times Y} f(g y) d \mu(g) d m(y) .
$$

We say that a measure $m$ is $\mu$-stationary if $\mu * m=m$.

$\mu$-boundary is a $G$-space equipped with a $\mu$-stationary probability measure $\lambda$ such that $\left(x_{n} \lambda\right)$ converges weakly to a $\delta$-measure for $\mathbb{P}$-almost all trajectory of the random walk $\left(x_{n}\right)$ associated with $(G, \mu)$.

The measure $\nu$ is $\mu$-stationary. If we assume in addition that $\mu$ has a finite first moment, that is to say,

$$
\sum_{g \in G} \mu(g) d(o, g o)<+\infty
$$

then $(\partial X, \nu)$ is in fact the Poisson boundary associated to $(G, \mu)([13])$, which means that every $\mu$-harmonic bounded function $f$ on $G$ can be written

$$
f(g)=\int_{\partial X} F(g \xi) d \nu(\xi)
$$

where $F$ is a bounded function on $\partial X$ (for details on this notion, see [8] and [14]).

A first result on the type of the harmonic measure is that under the hypotheses of Proposition 1.9 it doesn't have any atom. Besides, if the support of $\mu$ generates $G$ as a semi-group, then the support of the measure $\nu$ is the limit set $\Lambda_{G}$ of $G$. This is a consequence of the minimality of this limit set and of the stationarity of $\nu$, and can be established using Proposition 1.8, Proposition 1.9, and through the following lemma:

Lemma 1.10. Let $U$ be an open set in $\partial X$ which intersects $\Lambda_{G}$. Then $\nu(U)>0$.

Proof. Let $\gamma_{1}, \cdots, \gamma_{r} \in G$ be such that

$$
\Lambda_{G} \subset \bigcup_{1 \leq i \leq r} \gamma_{i} U
$$

and choose $s$ such that $\gamma_{1}, \cdots, \gamma_{r} \in \operatorname{supp}\left(\mu^{s}\right)$. Since $\nu$ is $\mu$-stationary we have

$$
\nu(U)=\sum_{g \in \operatorname{supp}\left(\mu^{s}\right)} \mu^{s}(g) \nu(g U) .
$$

Hence if $\nu(U)=0$, we would have $\nu\left(\Lambda_{G}\right)=0$. 
Asymptotic quantities. Let $\mu$ be a probability measure on $G$ having a finite first moment.

We write $|g|=d(o, g o)$, and for a probability measure $\lambda$,

$$
L(\lambda):=\sum_{g \in G} \lambda(g)|g| .
$$

Denote by $\mu^{n}$ the $n^{\text {th }}$ convolution of $\mu$, which is the distribution of the position at time $n$ of the random walk. The sequence $\left(L\left(\mu^{n}\right)\right)_{n}$ is subadditive, which allows us to adopt the following definition ([11]):

Definition 1.11. The limit of the sequence $\left(L\left(\mu^{n}\right) / n\right)_{n}$ is called the rate of escape of the random walk $\left(x_{n}\right)_{n \geq 0}$ and denoted by $l(G, \mu)$.

Moreover, $\mu$ having a finite first moment implies that the entropy

$$
H(\mu):=\sum_{g}-\mu(g) \log (\mu(g))
$$

of $\mu$ is finite. One can define in the same way the asymptotic entropy ([1]):

Definition 1.12. The limit of the sequence $\left(H\left(\mu^{n}\right) / n\right)_{n}$ is called the asymptotic entropy of the random walk $\left(x_{n}\right)_{n \geq 0}$ and denoted by $h(G, \mu)$.

Using Kingman's subadditive ergodic theorem, one gets ([] $)$ a $\mathbb{P}$-almost sure convergence:

$$
-\frac{1}{n} \log \left(\mu^{n}\left(x_{n}\right)\right) \longrightarrow h(G, \mu) \text { and } \frac{\left|x_{n}\right|}{n} \longrightarrow l(G, \mu) .
$$

If there is no ambiguity, we shall denote by $h$ and $l$ these two quantities. If in addition the support of $\mu$ generates $G$, both are positive (see [13]).

1.3. Dimension. Let $(Y, d)$ be a complete metric space. One defines the $\alpha$-Hausdorff measure of a set $Z \subset Y$ as

$$
m_{H}(Z, \alpha)=\lim _{\epsilon \rightarrow 0}\left(\inf \left\{\sum_{U \in \mathcal{G}_{\epsilon}}(\operatorname{diam}(U))^{\alpha}\right\}\right),
$$

where the infimum is taken over the covers $\mathcal{G}_{\epsilon}$ of $Z$ by open sets of diameter at most $\epsilon$. The usual Hausdorff dimension of $Z$ is then

$$
\operatorname{dim}_{H}(Z)=\inf \left\{\alpha: m_{H}(Z, \alpha)=0\right\}=\sup \left\{\alpha: m_{H}(Z, \alpha)=+\infty\right\} .
$$

When $m_{H}\left(Y, \operatorname{dim}_{H}(Y)\right)$ is finite and non-zero, the function $Z \mapsto m_{H}\left(Z, \operatorname{dim}_{H} Y\right)$ is, after normalization, a probability measure on $Y$, called the Hausdorff measure.

Dimensions of measures. Let $\nu$ be a probability measure on $(Y, d)$. We define the Hausdorff dimension of $\nu$ as

$$
\operatorname{dim}_{H} \nu=\inf \left\{\operatorname{dim}_{H} Z: \nu(Z)=1\right\} .
$$

Remark 1.13. The dimension of a measure characterizes to some extent its type. Indeed if $\lambda$ is absolutely continuous w.r.t. $\nu,\{Z: \nu(Z)=1\} \subset\{Z: \lambda(Z)=1\}$, so $\operatorname{dim}_{H} \lambda \leq \operatorname{dim}_{H} \nu$. 
To estimate the dimension of a set, and a fortiori of a measure, is usually not easy. We will have however a more direct way to estimate the dimension of a measure, introducing the (lower and upper) pointwise dimensions at a point $x$ :

$$
\underline{\operatorname{dim}}_{P} \nu(x)=\liminf _{r \rightarrow 0} \frac{\log \nu B(x, r)}{\log r} \text { and } \overline{\operatorname{dim}}_{P} \nu(x)=\limsup _{r \rightarrow 0} \frac{\log \nu B(x, r)}{\log r} .
$$

Moreover this notion allows a more intuitive vision of the dimension of a measure: it can be regarded as the rate of decrease of the measure of balls. To relate the Hausdorff and pointwise dimensions, we will need a condition on the space (see 21, appendix 1).

Definition 1.14. A metric space $(Y, d)$ is said to have finite multiplicity if there exists $K>0$ and $\epsilon_{0}>0$ such that for every $\left.\epsilon \in\right] 0, \epsilon_{0}[$, there exists a cover of multiplicity $K$ (i.e., in which every point belongs to at most $K$ balls) of $Y$ by balls of radius $\epsilon$.

Proposition 1.15 (see [21, appendix 1). Let $(Y, d)$ be a finite multiplicity space, and let $\nu$ be a probability measure on $Y$. If there exists a constant $d$ such that $\overline{\operatorname{dim}}_{P} \nu(x) \leq d \nu$-almost surely, then $\operatorname{dim}_{H} \nu \leq d$.

If $\underline{\operatorname{dim}}_{P} \nu(x) \geq d \nu$-almost surely, we also have $\operatorname{dim}_{H} \nu \geq d$. A probability measure with a constant $d$ such that $\underline{\operatorname{dim}}_{P} \nu(x)=\overline{\operatorname{dim}}_{P} \nu(x)=d \nu$-almost surely is said to be exact dimensional.

Case of the boundary of a hyperbolic group. We come back to our hyperbolic space $(X, d)$. As we want to consider the question of the maximality of the dimension of the harmonic measure, it is natural to ask about the dimension of the limit set $\Lambda_{G}$, which supports this measure. We define the growth (of base $a$ ) of $G$ by

$$
v_{a}(G)=\limsup _{R \rightarrow \infty} \frac{\log _{a} \operatorname{card}\{g \in G: d(o, g o) \leq R\}}{R} .
$$

Proposition 1.16 (4]). If in addition $G$ is quasi-convex-cocompact (see [4 for a definition), then $\Lambda_{G}$ has Hausdorff dimension $v_{a}(G)$ (with respect to the metric $\left.d_{a}\right)$.

We don't want to explore this hypothesis of quasi-convex-cocompactness. We just note that in the case of a discrete group acting on the hyperbolic half-plane it coincides with the notion of convex-cocompactness; and we shall also use the fact that a hyperbolic group $G$ acting on its Cayley graph has this property (4]).

In Section 2, we bound from above the pointwise dimension of the harmonic measure. In order to obtain a result on the singularity of this measure with respect to the $v_{a}(G)$-Hausdorff measure, we need, in view of Proposition 1.15] and Remark 1.13, a finite multiplicity result on $\left(\partial X, d_{a}\right)$. Let us remark that when $X$ is a tree, this is obvious because balls of the same diameter form a partition.

Theorem 1.17. Assume that $G$ is a hyperbolic group ( $X$ its Cayley graph). Then its hyperbolic boundary $\partial G$, equipped with the metric $d_{a}$, is of finite multiplicity.

Proof. We first note that if two geodesic rays joining $o$ with two points $\xi_{1}$ and $\xi_{2}$ of the boundary go through a same point $x$ such that $d(o, x)=n$, then $\left(\xi_{1} \mid \xi_{2}\right) \geq n-2 \delta$ and so (see Proposition 1.1) $d_{a}\left(\xi_{1}, \xi_{2}\right) \leq \lambda a^{-(n-2 \delta)}$. Denote by $W_{n}$ the set of words $w$ of length $n$ through which goes a certain geodesic ray starting at $o$; and for each 
$w$ in $W_{n}$ let $\xi_{w}$ be the limit point of such a ray. In view of our first remark, the set of all open balls $B\left(\xi_{w}, \lambda a^{-(n-2 \delta-1)}\right), w$ in $W_{n}$, is a cover of the boundary.

We are now going to show that these covers are of finite multiplicity (uniformly bounded in $n)$. Take $n>0$ and set $\epsilon=\lambda a^{-(n-2 \delta-1)}$. Let $\xi$ be a point in $\partial X$ and let $w$ be a word of length $n$ through which goes a ray $\left[o, \xi\left[\right.\right.$. Now let $w^{\prime}$ be an element in $W_{n}$ such that $B\left(\xi_{w^{\prime}}, \epsilon\right)$ is a ball of our cover in which $\xi$ lies. Since $d_{a}\left(\xi, \xi_{w^{\prime}}\right)<\epsilon$ and in view of Proposition 1.1, we have $\lambda^{-1} a^{-\left(\xi \mid \xi_{w^{\prime}}\right)} \leq \epsilon$, and so

$$
\left(\xi \mid \xi_{w^{\prime}}\right) \geq n-2 \delta-1-2 \log _{a} \lambda .
$$

But we have

$$
\left(w \mid w^{\prime}\right) \geq \min \left\{(w \mid \xi),\left(\xi \mid \xi_{w^{\prime}}\right),\left(\xi_{w^{\prime}} \mid w^{\prime}\right)\right\}-2 \delta,
$$

and, as a consequence of inequality (1.2), $(w \mid \xi) \geq n-2 \delta$ and $\left(\xi_{w^{\prime}} \mid w^{\prime}\right) \geq n-2 \delta$. This yields

$$
\begin{aligned}
\left(w \mid w^{\prime}\right) & \geq \min \left\{n-2 \delta,\left(\xi \mid \xi_{w^{\prime}}\right)\right\}-2 \delta \\
& \geq n-1-2 \log _{a} \lambda-4 \delta .
\end{aligned}
$$

Since $\left(w \mid w^{\prime}\right)=n-\frac{1}{2} d\left(w, w^{\prime}\right)$, we deduce that

$$
d\left(w, w^{\prime}\right) \leq 2+4 \log _{a} \lambda+8 \delta .
$$

But $G$ is finitely generated, so the number of $w^{\prime}$ in $W_{n}$ for which the distance from $w$ is less than this constant is bounded by a constant which doesn't depends on the length $n$ of $w$.

\section{Relation Between ASYMPtotic QUANTITIES AND POINTWise Dimension OF THE HARMONIC MEASURE}

We fix in this section a measure $\mu$ on $G$ with a finite first moment, and such that its support generates $G$ as a semigroup. We denote as in Section 1 by $\nu$ the distribution of $x_{\infty}$ on $\partial X$.

We have (see Section 1.2$) \mathbb{P}$-almost surely

$$
\frac{\left(x_{n-1} \mid x_{n}\right)}{n} \longrightarrow l \text { and }-\frac{\log \mu^{n}\left(x_{n}\right)}{n} \longrightarrow h .
$$

We define for $\epsilon>0$ and $N$ the set $\Omega_{\epsilon}^{N}$ of trajectories such that for $n \geq N$,

- $\left(x_{n-1} \mid x_{n}\right)>(l-\epsilon) n$,

- $-\log \mu^{n}\left(x_{n}\right)<(h+\epsilon) n$.

For $\eta>0$ there exists then an integer $N_{\epsilon, \eta}$ such that

$$
\forall N \geq N_{\epsilon, \eta}, \mathbb{P}\left(\Omega_{\epsilon}^{N}\right)>1-\eta .
$$

We write $\Omega_{\epsilon, \eta}=\Omega_{\epsilon}^{N_{\epsilon, \eta}}$. Denote also by $C_{\boldsymbol{x}}^{n}$ the set of trajectories whose $n^{\text {th }}$ position coincides with $x_{n}$. Our proof is based on the following lemma:

Lemma 2.1 ([12]). There exists a set $\Lambda_{\epsilon, \eta} \subset \Omega_{\epsilon, \eta}$ with measure greater than $1-2 \eta$, on which the quantity

$$
\frac{\mathbb{P}\left(C_{\boldsymbol{x}}^{n} \cap \Omega_{\epsilon, \eta}\right)}{\mu^{n}\left(x_{n}\right)}
$$

admits a strictly positive limit when $n$ goes to infinity. In particular on this set we have

$$
\limsup _{n} \frac{\log \mathbb{P}\left(C_{x}^{n} \cap \Omega_{\epsilon, \eta}\right)}{n}=\limsup _{n} \frac{\log \mu^{n}\left(x_{n}\right)}{n} .
$$


We write, for $\xi \in \partial X$ and $r>0$,

$$
D(\xi, r)=\left\{\boldsymbol{x}: x_{\infty} \in B(\xi, r)\right\} .
$$

In the next lemma we prove that if a trajectory in $\Omega_{\epsilon, \eta}$ coincides at time $n$ with $x_{n}$, then its endpoint is not too far from $x_{\infty}$.

Lemma 2.2. Fix two strictly positive numbers $\eta$ and $\epsilon$. We have, for $\boldsymbol{x} \in \Omega_{\epsilon, \eta}$ and $n \geq N_{\epsilon, \eta}$,

$$
C_{\boldsymbol{x}}^{n} \cap \Omega_{\epsilon, \eta} \subset D\left(x_{\infty}, \frac{2 \lambda a^{-(l-\epsilon)(n+1)}}{1-a^{-(l-\epsilon)}}\right),
$$

where $\lambda$ is the constant introduced in Proposition 1.1.

Proof. We fix $\boldsymbol{x} \in \Omega_{\epsilon, \eta}$. Let $\boldsymbol{x}^{\prime}$ be an element of $C_{\boldsymbol{x}}^{n} \cap \Omega_{\epsilon, \eta}$. Using the fact that $\boldsymbol{x}^{\prime} \in \Omega_{\epsilon, \eta}$, we have, for $n$ large enough, $\left(x_{n-1}^{\prime} \mid x_{n}^{\prime}\right)^{x}>(l-\epsilon) n$; and thus, using Proposition 1.1.

$$
d_{a}\left(x_{n-1}^{\prime}, x_{n}^{\prime}\right) \leq \lambda a^{-n(l-\epsilon)} .
$$

So for $m>n$,

which yields

$$
d_{a}\left(x_{n}^{\prime}, x_{m}^{\prime}\right) \leq \lambda a^{-(n+1)(l-\epsilon)} \sum_{k=0}^{m-1} a^{-k(l-\epsilon)},
$$

$$
d_{a}\left(x_{n}^{\prime}, x_{\infty}^{\prime}\right) \leq \frac{\lambda a^{-(n+1)(l-\epsilon)}}{1-a^{-(l-\epsilon)}} .
$$

Since $\boldsymbol{x}$ belongs to $C_{\boldsymbol{x}}^{n} \cap \Omega_{\epsilon, \eta}$, we get

$$
d_{a}\left(x_{\infty}, x_{\infty}^{\prime}\right) \leq \frac{2 \lambda a^{-(n+1)(l-\epsilon)}}{1-a^{-(l-\epsilon)}} .
$$

This lemma gives us a bound from below of the $\nu$-measure of the balls in $\partial X$ and so we obtain:

Proposition 2.3. For $\nu$-almost every $\xi$,

$$
\overline{\operatorname{dim}}_{P} \nu(\xi) \leq \frac{1}{\log a} \frac{h}{l} .
$$

Proof. Let $\boldsymbol{x}$ be an element in $\Lambda_{\epsilon, \eta}$. We have

$$
\overline{\operatorname{dim}}_{P} \nu\left(x_{\infty}\right)=\limsup _{r \rightarrow 0} \frac{\log \nu B\left(x_{\infty}, r\right)}{\log r}=\limsup _{r \rightarrow 0} \frac{\log \mathbb{P} D\left(x_{\infty}, r\right)}{\log r},
$$

and replacing $r$ by $\frac{2 \lambda a^{-(n+1)(l-\epsilon)}}{1-a^{-(l-\epsilon)}}$,

$$
\overline{\operatorname{dim}}_{P} \nu\left(x_{\infty}\right)=\limsup _{n \rightarrow \infty} \frac{\log \mathbb{P} D\left(x_{\infty}, \frac{2 \lambda a^{-(l-\epsilon)(n+1)}}{1-a^{-(l-\epsilon)}}\right)}{-\log a(n+1)(l-\epsilon)} .
$$

As $x \in \Omega_{\epsilon, \eta}$ we can use Lemma 2.2. which yields

$$
\overline{\operatorname{dim}}_{P} \nu\left(x_{\infty}\right) \leq \limsup _{n \rightarrow \infty} \frac{\log \mathbb{P}\left(C_{\boldsymbol{x}}^{n} \cap \Omega_{\epsilon, \eta}\right)}{-\log a(n+1)(l-\epsilon)},
$$

and then using Lemma 2.1 we get

$$
\overline{\operatorname{dim}}_{P} \nu\left(x_{\infty}\right) \leq \limsup _{n \rightarrow \infty} \frac{-\log \mu^{n}\left(x_{n}\right)}{\log a(n+1)(l-\epsilon)}=\frac{h}{\log a(l-\epsilon)} .
$$


Since this is true for each $\epsilon>0$ on a set of measure $1-2 \eta$ for each $\eta>0$, the proof is complete.

\section{An INTEgral FORMUla FOR THE RATE OF ESCAPE}

3.1. Busemann functions. We recall the definition of Busemann functions, which we will use to estimate the rate of escape. Let $\sigma$ be a geodesic ray. For each $x$ in $X$, the function $t \mapsto[d(x, \sigma(t))-t]$ is decreasing and bounded (it's just a consequence of the triangle inequality). So we can define the Busemann function associated to a geodesic ray $\sigma$ as

$$
f_{\sigma}(x)=\lim _{t \rightarrow \infty} d(x, \sigma(t))-t .
$$

Then we define a cocycle on $X^{2}$ by

$$
\beta_{\sigma}(x, y)=f_{\sigma}(y)-f_{\sigma}(x) .
$$

Remark 3.1. Assume in addition that the space $X$ has the following property $(P)$ : for two equivalent rays $\sigma_{1}$ and $\sigma_{2}$ (see Section 11 for the definition), there exists $T_{0}$ such that $\lim _{t \rightarrow \infty} d\left(\sigma_{1}(t), \sigma_{2}\left(t+T_{0}\right)\right)=0$. Under this assumption, if $\sigma_{1} \sim \sigma_{2}$, then we have

$$
\beta_{\sigma_{1}}(x, y)=\beta_{\sigma_{2}}(x, y) .
$$

So the function $\beta_{\sigma}$ depends only on the endpoint of the ray $\sigma$. This allows us to define a cocycle on $\partial X$, called the Busemann cocycle, by

$$
\beta_{\xi}(x, y)=\beta_{\sigma}(x, y)
$$

$\sigma$ being a ray with endpoint $\xi$.

The hyperbolic half-plane $\mathbb{H}^{2}$, trees, and more generally CAT $(-1)$ spaces have this property.

In the general case (not assuming that $X$ has property $(P)$ ) this construction fails because if two geodesic rays $\sigma_{1}$ and $\sigma_{2}$ have the same endpoint $\xi$, which means that there exists a constant $D$ such that for all $t, d\left(\sigma_{1}(t), \sigma_{2}(t)\right) \leq D$, then the equality (3.1) is not satisfied, but we only have

$$
\left|\beta_{\sigma_{1}}(x, y)-\beta_{\sigma_{2}}(x, y)\right| \leq 2 D .
$$

However, two geodesic rays with the same point at infinity have the following property: there exists $T_{0}$ such that $\lim _{t \rightarrow \infty} d\left(\sigma_{1}(t), \sigma_{2}\left(t+T_{0}\right)\right) \leq 16 \delta(\underline{9})$. This implies the following.

Lemma 3.2. Let $\sigma_{1}$ and $\sigma_{2}$ be two geodesic rays with the same point at infinity $\xi$. Then for any $x$ and $y$ in $X$, we have

$$
\left|\beta_{\sigma_{1}}(x, y)-\beta_{\sigma_{2}}(x, y)\right| \leq C_{1},
$$

where $C_{1}$ is a constant which depends only on $\delta$.

This allows us to adopt the following definition: for $\xi$ in $\partial X$ and $x, y$ in $X$,

$$
\beta_{\xi}(x, y)=\sup \left\{\beta_{\sigma}(x, y)\right\},
$$

where the supremum is taken over all rays with endpoint $\xi$. In particular, if $\sigma$ is a geodesic ray such that $\sigma(\infty)=\xi$, then

$$
\left|\beta_{\xi}(x, y)-\beta_{\sigma}(x, y)\right| \leq C_{1} \text {. }
$$


This will not be a cocycle, but we have

$$
\left|\beta_{\xi}(x, y)-\left(\beta_{\xi}(x, z)+\beta_{\xi}(z, y)\right)\right| \leq 3 C_{1} .
$$

We will need the following lemma (4):

Lemma 3.3. Let $\xi$ be a point in $\partial X, \sigma$ be a ray with endpoint $\xi$, and $x_{1}, x_{2}$ be two points in $X$. Then there exists a neighborhood $V$ of $\xi$ in $X \cup \partial X$ such that if $y \in X \cap V$,

$$
\left|\beta_{\sigma}\left(x_{1}, x_{2}\right)-\left(d\left(x_{2}, y\right)-d\left(x_{1}, y\right)\right)\right| \leq C^{\prime},
$$

where $C^{\prime}$ is a constant which depends only on $\delta$. In particular we have

$$
\left|\beta_{\xi}\left(x_{1}, x_{2}\right)-\left(d\left(x_{2}, y\right)-d\left(x_{1}, y\right)\right)\right| \leq C^{\prime}+C_{1}=C_{2} .
$$

3.2. Rate of escape. We shall get a formula for the rate of escape of a random walk in terms of this Busemann "quasi-cocycle".

Proposition 3.4. Let $\mu$ be a measure supported by $G$, having a finite first moment, and whose support generates $G$. We denote as in Section 1 by $\nu$ the distribution of $x_{\infty}$ on $\partial X$. We then have the following estimate of the rate of escape:

$$
\left|l(G, \mu)-\sum_{g} \mu(g) \int_{\partial X} \beta_{\xi}\left(o, g^{-1} o\right) d \nu(\xi)\right| \leq C_{2},
$$

where $C_{2}$ is the constant introduced in Lemma 3.3 .

Proof. Writing

$$
L_{n}=\int d\left(o, x_{n} o\right) d \mathbb{P},
$$

we have by definition

$$
l(G, \mu)=\lim _{n} \frac{L_{n}}{n} .
$$

So we have

$$
\liminf _{n}\left(L_{n+1}-L_{n}\right) \leq l(G, \mu) \leq \limsup _{n}\left(L_{n+1}-L_{n}\right) .
$$

Besides we have

$$
\begin{aligned}
L_{n+1} & =\int_{G} d(o, \gamma o) d \mu^{n+1}(\gamma) \\
& =\sum_{g} \mu(g) \int d(o, g \gamma o) d \mu^{n}(\gamma) \\
& =\sum_{g} \mu(g) \int d\left(o, g x_{n} o\right) d \mathbb{P},
\end{aligned}
$$

and so

$$
\begin{aligned}
L_{n+1}-L_{n} & =\sum_{g} \mu(g) \int\left(d\left(o, g x_{n} o\right)-d\left(o, x_{n} o\right)\right) d \mathbb{P} \\
& =\sum_{g} \mu(g) \int\left(d\left(g^{-1} o, x_{n} o\right)-d\left(o, x_{n} o\right)\right) d \mathbb{P} .
\end{aligned}
$$


The quantity $\left(d\left(g^{-1} o, x_{n} o\right)-d\left(o, x_{n} o\right)\right)$ is bounded for every $n$ by $d(o, g o)$, which is an integrable function w.r.t. $\mu \otimes \mathbb{P}$; hence we can apply the Lebesgue convergence theorem, which implies:

$$
\liminf _{n}\left(L_{n+1}-L_{n}\right) \geq \sum_{g} \mu(g) \int \liminf _{n}\left(d\left(g^{-1} o, x_{n} o\right)-d\left(o, x_{n} o\right)\right) d \mathbb{P}
$$

Moreover Lemma 3.3 gives

$$
\left|\liminf _{n}\left(d\left(g^{-1} o, x_{n} o\right)-d\left(o, x_{n} o\right)\right)-\beta_{x_{\infty}}\left(o, g^{-1} o\right)\right| \leq C_{2},
$$

from which we deduce

$$
l(G, \mu) \geq \sum_{g} \mu(g) \int \beta_{x_{\infty}}\left(o, g^{-1} o\right) d \mathbb{P}-C_{2} .
$$

The same arguments apply for the upper limit.

Remark 3.5. If $(X, d)$ has property $(P)$ (see Remark 3.1) the formula of Proposition 3.4 becomes an equality:

$$
l(G, \mu)=\sum_{g} \mu(g) \int_{\partial X} \beta_{\xi}\left(o, g^{-1} o\right) d \nu(\xi) .
$$

\section{Construction}

Our aim in this section is to construct a random walk such that the associated ratio $h / l$ is arbitrarily small. For this purpose, we are going to construct a sequence $\left(\mu_{k}\right)$ of probability measures such that

$$
\lim _{k \rightarrow \infty} \frac{h\left(G, \mu_{k}\right)}{l\left(G, \mu_{k}\right)}=0 .
$$

4.1. Introduction of the sequence $\left(\mu_{k}\right)$. Let us fix a measure $\mu$ on $G$ with a finite first moment, and whose support generates $G$ as a semigroup. Let $\gamma_{0}$ be a hyperbolic element (see Definition [1.4) in $G$; we write $\gamma_{k}=\gamma_{0}^{k} o$ and $\gamma_{ \pm}=\lim _{k \rightarrow \pm \infty} \gamma_{k}$. Then for each $k \geq 0$ we take

$$
\mu_{k}=\frac{1}{2} \mu+\frac{1}{4}\left(\delta_{\gamma_{0}^{k}}+\delta_{\gamma_{0}^{-k}}\right)
$$

Remark that each $\mu_{k}$ satisfies the hypotheses of Proposition 1.9. We denote by $\nu_{k}$ the harmonic measure associated with each $\mu_{k}$.

Proposition 4.1. The quantity $h\left(G, \mu_{k}\right)$ is bounded by a constant which doesn't depend on $k$.

Proof. We know that $h\left(G, \mu_{k}\right)$ is bounded from above by $H\left(\mu_{k}\right)$ and we have

$$
\begin{aligned}
H\left(\mu_{k}\right)= & -\frac{1}{2} \sum_{g} \mu(g) \log \left(\frac{1}{2} \mu(g)+\frac{1}{4}\left(\delta_{\gamma_{k}}(g)+\delta_{\gamma_{-k}}(g)\right)\right) \\
& -\frac{1}{4} \log \left(\frac{1}{2} \mu\left(\gamma_{k}\right)+\frac{1}{4}\right)-\frac{1}{4} \log \left(\frac{1}{2} \mu\left(\gamma_{-k}\right)+\frac{1}{4}\right) \\
\leq & -\frac{1}{2} \sum_{g} \mu(g) \log \left(\frac{1}{2} \mu(g)\right)-\frac{1}{2} \log \left(\frac{1}{4}\right) \leq \frac{3 \log 2}{2}+\frac{1}{2} H(\mu) .
\end{aligned}
$$


Hence it just remains to show that $l\left(G, \mu_{k}\right)$ goes to infinity. By using the formula of Proposition 3.4 we get

$$
l\left(G, \mu_{k}\right) \geq \sum_{g} \mu_{k}(g) \int_{\partial X} \beta_{\xi}\left(o, g^{-1} o\right) d \nu_{k}(\xi)-C_{2} .
$$

But

$$
\begin{aligned}
\sum_{g} \mu_{k}(g) \int_{\partial X} \beta_{\xi}\left(o, g^{-1} o\right) d \nu_{k}(\xi)=\frac{1}{2} \sum_{g} & \mu(g) \int_{\partial X} \beta_{\xi}\left(o, g^{-1} o\right) d \nu_{k}(\xi) \\
& +\frac{1}{4} \int_{\partial X}\left[\beta_{\xi}\left(o, \gamma_{k}\right)+\beta_{\xi}\left(o, \gamma_{-k}\right)\right] d \nu_{k}(\xi)
\end{aligned}
$$

and since $\left|\beta_{\xi}\left(o, g^{-1} o\right)\right| \leq d(o, g o)$, the first element in this sum is bounded in absolute value by $L(\mu) / 2$. Then, writing $C_{3}=C_{2}+L(\mu) / 2$, we get

$$
l\left(G, \mu_{k}\right) \geq \frac{1}{4} \int_{\partial X}\left[\beta_{\xi}\left(o, \gamma_{k}\right)+\beta_{\xi}\left(o, \gamma_{-k}\right)\right] d \nu_{k}(\xi)-C_{3} .
$$

We are now going to estimate the quantity in the square brackets in the previous equation.

4.2. Estimate of $\left[\beta_{\xi}\left(o, \gamma_{k}\right)+\beta_{\xi}\left(o, \gamma_{-k}\right)\right]$. We first show a convexity inequality based on the following property of hyperbolic spaces.

Proposition 4.2 (see [5]). In the $\delta$-hyperbolic space $(X, d)$, the metric has the following quasi-convexity property: let $x_{1}$ and $x_{2}$ be two points in $X$ and $s:[0,1] \rightarrow X$ a constant speed parametrization of a geodesic segment joining $x_{1}$ and $x_{2}$. If $y$ is another point in $X$, then we have, for all $t$ in $[0,1]$,

$$
d(y, s(t)) \leq t d\left(y, x_{1}\right)+(1-t) d\left(y, x_{2}\right)+4 \delta .
$$

We deduce the following property:

Proposition 4.3. There exists a constant $C_{4}$ which depends only on $\delta$ and $\gamma_{0}$ such that for every $k \geq 0$ and $\xi \in \partial X$,

$$
\beta_{\xi}\left(o, \gamma_{k}\right)+\beta_{\xi}\left(o, \gamma_{-k}\right) \geq-C_{4} .
$$

Proof. Let $\sigma$ be a geodesic joining $\gamma_{+}$and $\gamma_{-}, K$ the Hausdorff distance between $\sigma$ and the quasi-geodesic associated with $\gamma_{0}$ (see Proposition 1.5). Choose $o^{\prime}$ on $\sigma$ such that $d\left(o, o^{\prime}\right) \leq K+1, \gamma_{ \pm k}^{\prime}$ on $\sigma$ such that $d\left(\gamma_{ \pm k}, \gamma_{ \pm k}^{\prime}\right) \leq K+1$. Denote by $m_{k}^{\prime}$ the middle point of the segment $\left[\gamma_{k}^{\prime}, \gamma_{-k}^{\prime}\right]$ (see figure below).

For each $y$ in $X$ we have

$$
\begin{aligned}
d\left(y, \gamma_{k}\right)-d(y, o) & +d\left(y, \gamma_{-k}\right)-d(y, o) \\
& \geq d\left(y, \gamma_{k}^{\prime}\right)-d\left(y, o^{\prime}\right)+d\left(y, \gamma_{-k}^{\prime}\right)-d\left(y, o^{\prime}\right)-4(K+1),
\end{aligned}
$$

and

$d\left(y, \gamma_{k}^{\prime}\right)-d\left(y, o^{\prime}\right)+d\left(y, \gamma_{-k}^{\prime}\right)-d\left(y, o^{\prime}\right) \geq d\left(y, \gamma_{k}^{\prime}\right)+d\left(y, \gamma_{-k}^{\prime}\right)-2 d\left(y, m_{k}^{\prime}\right)-2 d\left(o^{\prime}, m_{k}^{\prime}\right)$.

But using the convexity property of the distance we get

$$
d\left(y, \gamma_{k}^{\prime}\right)+d\left(y, \gamma_{-k}^{\prime}\right)-2 d\left(y, m_{k}^{\prime}\right) \geq-8 \delta,
$$




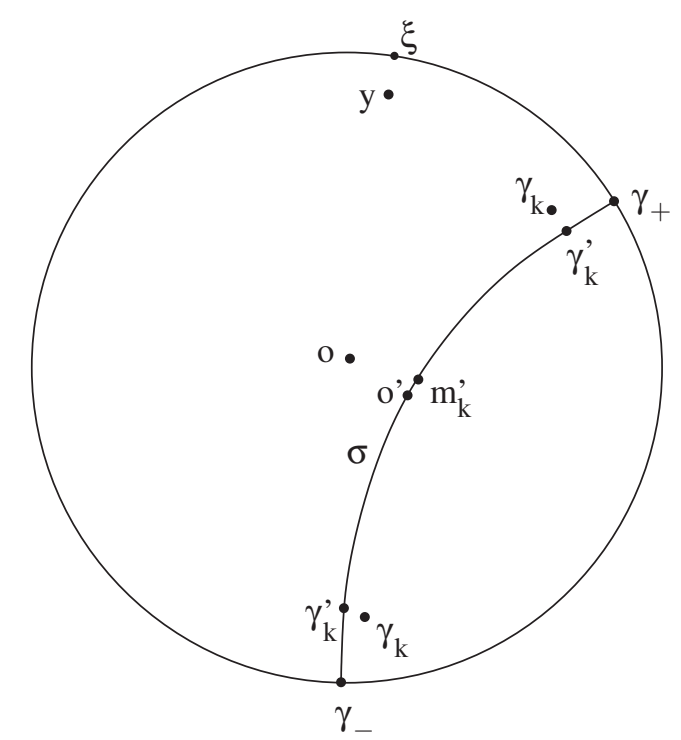

and since $d\left(o, \gamma_{k}\right)=d\left(o, \gamma_{-k}\right)$,

$$
\begin{aligned}
\left|d\left(o^{\prime}, \gamma_{k}^{\prime}\right)-d\left(o^{\prime}, \gamma_{-k}^{\prime}\right)\right|= & \mid\left[d\left(o^{\prime}, \gamma_{k}^{\prime}\right)-d\left(o, \gamma_{k}^{\prime}\right)+d\left(o, \gamma_{k}^{\prime}\right)-d\left(o, \gamma_{k}\right)\right] \\
& -\left[d\left(o^{\prime}, \gamma_{-k}^{\prime}\right)-d\left(o, \gamma_{-k}^{\prime}\right)+d\left(o, \gamma_{-k}^{\prime}\right)-d\left(o, \gamma_{-k}\right)\right] \mid \\
\leq & 2 d\left(o, o^{\prime}\right)+d\left(\gamma_{k}^{\prime}, \gamma_{k}\right)+d\left(\gamma_{-k}^{\prime}, \gamma_{-k}\right) \leq 4(K+1),
\end{aligned}
$$

which yields

$$
d\left(o^{\prime}, m_{k}^{\prime}\right) \leq 2(K+1) .
$$

So we get

$$
d\left(y, \gamma_{k}\right)-d(y, o)+d\left(y, \gamma_{-k}\right)-d(y, o) \geq-4(K+1)-8 \delta-4(K+1) .
$$

Now Lemma 3.3 allows us to complete the proof, denoting $8(K+1)+8 \delta+C_{2}$ by $C_{4}$.

We are now going to show that on a "big enough" subset (w.r.t. the measures $\left.\nu_{k}\right)$ of the boundary, the quantity $\left[\beta_{\xi}\left(o, \gamma_{k}\right)+\beta_{\xi}\left(o, \gamma_{-k}\right)\right]$ goes to $+\infty$ when $k$ does. We have, if $y$ is in a neigbourhood of $\xi$, again using Lemma 3.3.

$$
\beta_{\xi}\left(o, \gamma_{k}\right)+\beta_{\xi}\left(o, \gamma_{-k}\right) \geq d\left(y, \gamma_{k}\right)-d(y, o)+d\left(y, \gamma_{-k}\right)-d(y, o)-C_{2} .
$$

Hence

$$
\beta_{\xi}\left(o, \gamma_{k}\right)+\beta_{\xi}\left(o, \gamma_{-k}\right) \geq d\left(o, \gamma_{k}\right)+d\left(o, \gamma_{-k}\right)-2\left(y \mid \gamma_{k}\right)-2\left(y \mid \gamma_{-k}\right)-C_{2},
$$

so if the two Gromov products in this sum are bounded (which is the case if $\xi \notin$ $\left.\left\{\gamma_{+}, \gamma_{-}\right\}\right)$, then we have

$$
\lim _{k \rightarrow \infty} \beta_{\xi}\left(o, \gamma_{k}\right)+\beta_{\xi}\left(o, \gamma_{-k}\right)=+\infty .
$$

However, in order to complete the proof, using formula (4.1), we need that the quantity $\left[\beta_{\xi}\left(o, \gamma_{k}\right)+\beta_{\xi}\left(o, \gamma_{-k}\right)\right]$ goes to infinity uniformly on a set whose $\nu_{k}$-measure remains greater than a strictly positive constant. 
Lemma 4.4. Let $U$ be a neigbourhood of $\gamma_{+}$in $\partial X$. Then there exists a constant $C>0$ and an integer $K$ such that for every $k \geq K$ and every $\xi \notin U$,

$$
\left(\xi \mid \gamma_{k}\right) \leq C
$$

Proof. We saw that the topology on $\partial X$ was defined by neighborhoods of the type $\left\{\xi^{\prime}:\left(\xi \mid \xi^{\prime}\right) \geq D^{\prime}\right\}$. Let $C^{\prime}$ be a constant such that if $\left(\xi \mid \gamma_{+}\right) \geq C^{\prime}$, then $\xi \in U$. Take a $K$ such that if $k \geq K$, then $\left(\gamma_{k} \mid \gamma_{+}\right) \geq C^{\prime}+\delta$. Now let $\xi$ be an element of $\partial X \backslash U$. If we had $\left(\gamma_{k} \mid \xi\right) \geq C^{\prime}+\delta$, we would have

$$
\left(\xi \mid \gamma_{+}\right) \geq \min \left\{\left(\gamma_{k} \mid \gamma_{+}\right),\left(\gamma_{k} \mid \xi\right)\right\}-\delta \geq C^{\prime} .
$$

So we deduce the result by taking $C=C^{\prime}+\delta$.

We saw in Lemma 1.10 how to show that the harmonic measure of an open set was strictly positive by using the minimality of the limit set through Proposition 1.8. We are now going to show in the same way that we can bound from below uniformly w.r.t. $k$ the $\nu_{k}$-measure of an open set.

Proposition 4.5. Let $U$ be an open set meeting $\Lambda_{G}$. There exists a constant $\alpha>0$ (which depends on $U$ and $\mu$ ) such that for every $k$,

$$
\nu_{k}(U) \geq \alpha .
$$

Proof. We take $\gamma_{1}, \cdots, \gamma_{r} \in G$ as in Proposition 1.8.

$$
\Lambda_{G} \subset \bigcup_{1 \leq i \leq r} \gamma_{i} U,
$$

and $s$ such that $\gamma_{1}, \cdots, \gamma_{r} \in \operatorname{supp}\left(\mu^{s}\right)$. Now let us make the following remark: we have a kind of uniform stationarity of the $\nu_{k}$. Indeed as $\nu_{k}=\mu_{k} * \nu_{k}$ and, for every $k, \mu_{k} \geq 1 / 2 \mu$, we have

$$
\nu_{k} \geq \frac{1}{2^{s}} \mu^{s} * \nu_{k}
$$

and so

$$
\begin{aligned}
\nu_{k}(U) & \geq \frac{1}{2^{s}} \sum_{g \in \operatorname{supp}\left(\mu^{s}\right)} \mu^{s}(g) \nu_{k}(g U) \\
& \geq \frac{1}{2^{s}} \sum_{i} \mu^{s}\left(\gamma_{i}\right) \nu_{k}\left(\gamma_{i} U\right) \\
& \geq \frac{1}{2^{s}} \min _{i} \mu^{s}\left(\gamma_{i}\right) .
\end{aligned}
$$

Since $G$ is assumed to be non-elementary, $\Lambda_{G}$ contains an element which is distinct from $\gamma_{+}$and $\gamma_{-}$, so we can fix an open set $U$ meeting $\Lambda_{G}$ and which doesn't contain either $\gamma_{+}$or $\gamma_{-}$. Using Lemma 4.4 with $\partial X \backslash U$ as a neighborhood of $\gamma_{+}\left(\right.$and $\gamma_{-}$), we take $C_{5}$ and $K$ such that if $\xi \in U$ and $k \geq K$,

$$
\left(\xi \mid \gamma_{ \pm k}\right) \leq C_{5}
$$

Using formula (4.2), we get, for $k \geq K$ and $\xi \in U$ :

$$
\beta_{\xi}\left(o, \gamma_{k}\right)+\beta_{\xi}\left(o, \gamma_{-k}\right) \geq 2 d\left(o, \gamma_{k}\right)-4 C_{5}-C_{2} .
$$


Besides, formula (4.1) and Proposition 4.3 give us

$$
l\left(G, \mu_{k}\right) \geq \frac{1}{4} \int_{U}\left[\beta_{\xi}\left(o, \gamma_{k}\right)+\beta_{\xi}\left(o, \gamma_{-k}\right)\right] d \nu_{k}(\xi)-\frac{C_{4}}{4}+C_{3},
$$

and now we use the previous proposition to get

$$
l\left(G, \mu_{k}\right) \geq \frac{\alpha}{4}\left[2 d\left(o, \gamma_{k}\right)-4 C_{5}-C_{2}\right]-\frac{C_{4}}{4}-C_{3} .
$$

Since the first element of the right member in the previous equation goes to infinity and $h\left(G, \mu_{k}\right)$ is bounded, this gives us:

Proposition 4.6. With the notation previously adopted,

$$
\lim _{k \rightarrow \infty} \frac{h\left(G, \mu_{k}\right)}{l\left(G, \mu_{k}\right)}=0 \text {. }
$$

\section{MAin ReSUlt}

Putting together Proposition 4.6 and Proposition 2.3, we get the following result:

Theorem 5.1. Let $G$ be a subgroup of the group of isometries of a hyperbolic space $(X, d)$ acting properly discontinuously and which is not elementary. For every $\epsilon>0$ there exists on $G$ a symmetric probability measure $\mu$ with a finite first moment, whose support generates $G$, and which has the following property: the pointwise dimension of the harmonic measure $\nu$ associated with $(G, \mu)$ is $\nu$-almost surely smaller than $\epsilon$.

Remark 5.2. In the previous theorem, if moreover $G$ is finitely generated, $\mu$ can be chosen to be finitely supported.

Now we restrict ourselves to the case where $(X, d)$ is the Cayley graph of a hyperbolic group $G$. In this case, in view of Propositions 1.15] and 1.17. Theorem 5.1 implies that the Hausdorff dimension of the harmonic measure can be strictly lower than the dimension of the boundary, whose value is $v_{a}(G)$. In view of Remark 1.13. this implies that the harmonic and Hausdorff measures are not equivalent; since both are ergodic, they are singular. So we get the following result on the type of the harmonic measure:

Corollary 5.3. In the case where $X$ is the Cayley graph of a hyperbolic group $G$, there exists on $G$ a symmetric probability measure $\mu$ with a finite first moment, whose support generates $G$, and such that the associated harmonic measure and the Hausdorff measure on $\partial G$ are mutually singular.

Let us finally note that concerning the asymptotic behaviour of the sequence $\left(\nu_{k}\right)$, we can prove that for every subsequence of $\left(\nu_{k}\right)$ which converges weakly to a certain measure $\nu$, all points of the orbit of $\gamma_{+}$and $\gamma_{-}$under $G$ are atoms for $\nu$. In the case of a finitely generated free group, the sequence $\left(\nu_{k}\right)$ even converges weakly to an atomic measure supported by the orbits of $\gamma_{+}$and $\gamma_{-}$under $G$.

\section{ACKNOWLEDGMENTS}

I would like to thank my Ph.D. supervisor Vadim Kaimanovich for his advice during the redaction of this paper. 


\section{REFERENCES}

1. A. Avez, Entropie des groupes de type fini, C. R. Acad. Sci. Paris, Sér. A 275 (1972), pp. 241-270. MR0324741(48:3090)

2. S. D. Chatterji, Masse, die von regelmässigen Kettenbrüchen induziert sind, Math. Ann., vol. 164 (1966), pp. 113-117. MR0193079(33:1300)

3. M. Coornaert, Sur les groupes proprement discontinus d'isométries des espaces hyperboliques au sens de M. Gromov, Thèse, Strasbourg, 1990. MR1116319 (92i:57032)

4. M. Coornaert, Mesures de Patterson-Sullivan sur le bord d'un espace hyperbolique au sens de Gromov, Pacific Journal of Mathematics, vol.159 (1993), pp. 241-270, 1990. MR1214072 (94m:57075)

5. M. Coornaert, T. Delzant, A. Papadopoulos, Géométrie et théorie des groupes: les groupes hyperboliques de Gromov, Lecture Notes in Math. 1441, Springer, 1990. MR1075994 (92f:57003)

6. Y. Derriennic, Quelques applications du théorème ergodique sous-additif, Astérisque 74 (1980), pp. 183-201. MR0588163 (82e:60013)

7. E. B. Dynkin, M. B. Maljutov, Random walks on groups with a finite number of generators, Dokl. Akad. Nauk SSSR 137 (1961), pp. 1042-1045. MR0131904(24:A1751)

8. H. Furstenberg, Boundary theory and stochastic processes on homogeneous spaces, Proc. Symp. Pure Math., vol. 26 (1973), AMS, Providence R.I., pp. 193-229. MR0352328 (50:4815)

9. E. Ghys, P. De La Harpe (eds.), Sur les Groupes Hyperboliques d'après Mikhael Gromov, Birkhäuser, Basel, 1990. MR.1086648 (92f:53050)

10. M. Gromov, Hyperbolic groups, Essays in Group Theory (S.M. Gersten, ed.), MSRI Publ., vol. 8, Springer, New York, 1987, pp. 75-263. MR0919829 (89e:20070)

11. Y. Guivarc'h, Sur la loi des grands nombres et le rayon spectral d'une marche aléatoire, Astérisque 74 (1980), pp. 47-78. MR0588157(82g:60016)

12. V. A. Kaimanovich, Hausdorff dimension of the harmonic measure on trees, Ergod. Th. \& Dynam. Sys. (1998), pp. 631-660. MR1631732 (99g:60123)

13. V. A. Kaimanovich, The Poisson formula for groups with hyperbolic properties, Annals of Mathematics, vol. 152 (2000), pp. 659-692. MR.1815698(2002d:60064)

14. V. A. Kaimanovich, A. M. Vershik, Random walks on discrete groups : boundary and entropy, Ann. Prob. 11 (1983), pp. 457-490. MR0704539 (85d:60024)

15. Y. Kifer, F. Ledrappier, Hausdorff dimension of the harmonic measure on negatively curved manifolds, Trans. Amer. Math. Soc. 318 (1990), pp. 685-704. MR0951889 (91a:58205)

16. Y. Kifer, Y. Peres, B. Weiss, A dimension gap for continued fractions with independant digits, Israel J. of Math. 124 (2000), pp. 61-76. MR1856504 (2002m:11074)

17. F. Ledrappier, Quelques propriétés des exposants caractéristiques, Lecture Notes in Math., vol. 1097, Springer, Berlin, 1982. MR0876081 (88b:58081)

18. F. Ledrappier, Some asymptotic properties of random walks on free groups, in J. Taylor (ed), Topics in probability and Lie groups : boundary theory, CRM Proc. and Lecture Notes 28 (2001), AMS, pp. 117-152. MR1832436 (2002g:60116)

19. B. Levit, S. Molchanov, Invariant Markov chains on a free group with a finite number of generators, Vestnik Moskow Univ. 26(4) (1971), pp. 80-88. MR0298721 (45:7770)

20. J. Mairesse, F. Mathéus, Random walks on free products of cyclic groups and on Artin groups with two generators, preprint.

21. Ya. B. Pesin, Dimension theory in dynamical systems, Chicago Lect. Notes in Math. (1997). MR.1489237 (99b:58003)

22. F. Przytycki, M. Urbanski, A. Zdunik, Harmonic, Gibbs, and Hausdorff measures on repellers for holomorphic maps I, Ann. of Math. 130(1) (1989), pp. 1-40. MR1005606 (91i:58115)

23. A. M. Vershik, Dynamic theory of growth in groups : entropy, boundaries, examples, Russian Math. Surveys 55:4 (2000), pp. 667-733. MR1786730 (2001m:37019)

IRMar, Université de Rennes 1, Campus de Beaulieu, 35042 Rennes Cedex, France E-mail address: vincent.leprince@univ-rennes1.fr 